Accepted Manuscript

This is an Accepted Manuscript of an article published by Taylor \& Francis Group in Journal of Toxicology and Environmental Health, Part A: Current Issues on 29 August 2017, available online:

http://www.tandfonline.com/10.1080/15287394.2017.1352215.

You Song, Linn Mari Evenseth, Taisen Iguchi \& Knut Erik Tollefsen (2017) Release of chitobiase as an indicator of potential molting disruption in juvenile Daphnia magna exposed to the ecdysone receptor agonist 20hydroxyecdysone, Journal of Toxicology and Environmental Health, Part A, 80:16-18, 954-962.

It is recommended to use the published version for citation. 


\title{
Release of chitobiase as an indicator of potential molting disruption in juvenile Daphnia magna exposed to the ecdysone receptor agonist 20 -hydroxyecdysone
}

\author{
You Song ${ }^{*}, \mathrm{a}$, Linn Mari Evenseth ${ }^{\mathrm{b}}$, Taisen Iguchi ${ }^{\mathrm{c}, \mathrm{d}}$, Knut Erik Tollefsen ${ }^{\mathrm{a}, \mathrm{e}}$
}

${ }^{\text {a} N o r w e g i a n ~ I n s t i t u t e ~ f o r ~ W a t e r ~ R e s e a r c h ~(N I V A), ~ G a u s t a d a l l e ́ e n ~ 21, ~ N-0349 ~ O s l o, ~ N o r w a y ~}$

${ }^{b}$ Department of Medical Biology, Faculty of Health Sciences, University of Tromsø-The Arctic University of Norway, NO-9037 Tromsø, Norway

${ }^{\mathrm{c} D e p a r t m e n t ~ o f ~ B a s i c ~ B i o l o g y, ~ F a c u l t y ~ o f ~ L i f e ~ S c i e n c e, ~ S O K E N D A I, ~(G r a d u a t e ~ U n i v e r s i t y ~ f o r ~ A d v a n c e d ~}$ Studies), 5-1 Higashiyama, Myodaiji, Okazaki, Aichi 444-8787, Japan

${ }^{\mathrm{d} O k a z a k i}$ Institute for Integrative Bioscience, National Institute for Basic Biology (NIBB), National Institutes of Natural Sciences, 5-1 Higashiyama, Myodaiji, Okazaki, Aichi 444-8787, Japan

eNorwegian University of Life Sciences (NMBU), Faculty of Environmental Science and Technology, Department of Environmental Sciences (IMV), Centre for Environmental Radioactivity (CERAD). P.O. Box 5003, N-1432 Ås, Norway

*Corresponding author:

Name: You Song

Address: Norwegian Institute for Water Research (NIVA), Gaustadalléen 21, N-0349 Oslo, Norway

Phone: +4702348

Fax: +4722185200

E-mail address: you.song@niva.no 


\section{Abstract}

During arthropod molting, the old exoskeleton is degraded and recycled by the molting fluid. Chitobiase, a major chitinolytic enzyme in the molting fluid, has been widely used as a biomarker to indicate endocrine disruption of molting in arthropods under environmental stress. Although the release of chitobiase has been extensively studied in organisms exposed to molting-inhibiting chemicals, its association with molting and the response of the molting hormone receptor, ecdysone receptor (EcR), is not well understood. The present study was therefore conducted to identify potential linkages between the release of chitobiase, molting frequency and EcR activation in a freshwater crustacean Daphnia magna after short-term (96h) exposure to the endogenous molting hormone 20-hydroxyecdysone (20E). A suite of bioassays was used for this purpose, including the chitobiase activity assay, molting frequency assay, viability assay and in vitro EcR activation assay. Effect concentrations were compared between different assays analyzed. The results clearly showed that exposure to $20 \mathrm{E}$ reduced the chitobiase release and molting frequency in a concentration-dependent manner. Exposure to as low as $250 \mathrm{nM} 20 \mathrm{E}$ significantly reduced the release of chitobiase after $72 \mathrm{~h}$ exposure, whereas adverse effects on molting frequency and incomplete molting-associated mortality required higher exposure concentrations of $20 \mathrm{E}$ to occur. The EcR reporter assay further showed that as low as $100 \mathrm{nM} 20 \mathrm{E}$ may activate the EcR in vitro. The present study suggested that the release of chitobiase can be used as a sensitive indicator of potential molting disruption in crustaceans after exposure to EcR agonists such as $20 \mathrm{E}$. 


\section{Key words}

Endocrine disruption, Daphnia magna, Chitobiase, 20-Hydroxyecdysone, Ecdysone receptor, Molting

\section{Introduction}

Molting is an important biological process for growth, development and reproduction in arthropods and precisely regulated by complex neuroendocrine systems (Zitnan et al., 2007). During a molt cycle, a series of physiological and behavioral changes take place, such as formation of new cuticle, degradation and reuptake of old cuticle, shedding of old cuticle (ecdysis) and post-ecdysis cuticle tanning (Reynolds, 1987). The molting fluid is a cocktail of enzymes and inorganic ions involved in the degradation and recycling of old cuticles (Reynolds and Samuels, 1996). The molting fluid enzymes mainly include chitinolytic enzymes, such as chitinases and chitobiase ( $N$-acetyl- $\beta$-glucosaminidase), and various types of proteases (Reynolds and Samuels, 1996). The chitinolytic enzymes are responsible for degradation of cuticle chitin to $N$-acetyl- $\beta$-glucosamine (NAG) monomers, whereas the proteases break down the cuticle proteins to peptides and amino acids (Reynolds and Samuels, 1996; Merzendorfer and Zimoch, 2003). Controlled expression and secretion of molting fluid enzymes are vital to successful molting in arthropods.

One of the chitinolytic enzymes, chitobiase, has been widely used as a biomarker to study the effects of environmental stressors on molting, growth, biomass and population dynamics in arthropods (Espie and Roff, 1995; Sastri and Roff, 2000a; Zou, 2005; Richards et al., 2008; Conley et al., 2009; Duchet et al., 2011; Qi et al., 2013; de Souza Machado et al., 2017). 
Chitobiase is secreted in the epidermis and hepatopancreas (Spindler-Barth et al., 1990; Zou and Fingerman, 1999a) and released to the surrounding environment during molting (Espie and Roff, 1995). The expression of chitobiase is positively regulated by the endogenous molting hormone 20-hydroxyecdysone (20E) through transcriptional activation of the ecdysone receptor (EcR) (Zou and Fingerman, 1999b; Merzendorfer and Zimoch, 2003; Zheng et al., 2008), whereas its release to the environment is normally associated with the ecdysis behavior (Duchet et al., 2011). Therefore, both internal and ambient chitobiase activity may serve as indicators of the molting frequency and quality (Espie and Roff, 1995; Sastri and Roff, 2000b; Hanson and Lagadic, 2005).

Ecdysone receptor agonists are a group of chemicals that can activate the EcR and modulate molting signaling. Exposure to EcR agonists usually leads to delayed or precocious molting, most likely due to perturbation of ecdysis (Song et al., 2017). Based on this mode of action (MoA), various pesticides (e.g. tebufenozide, methoxyfenozide) have been developed against harmful arthropods (Nakagawa, 2005). In some cases, those EcR agonists may also affect nontarget species, such as aquatic crustaceans (e.g. shrimps, crabs and lobsters) which may have both ecological and economic values (De Wilde et al., 2013). Use of epidermal chitobiase mRNA expression and enzymatic activity has been proven to be reliable indicators of EcR activation by EcR agonists (Zou and Fingerman, 1999b; Meng and Zou, 2009). Since the release of chitobiase to the environment is usually associated with the cuticle shedding behavior, it may also become a non-invasive and integrated indicator of molting and disruption of this key step in arthropod development.

The next generation hazard assessment of chemicals requires enhanced predictability across 
chemicals and taxa, better understanding and extrapolation between different biological levels of organization, increased use of alternative approaches such as in vitro screening and in silico modeling, and reduced animal tests (NRC, 2007). The introduction of the adverse outcome pathway (AOP) concept complies with these new strategies by developing a conceptual framework to aggregate, evaluate and visualize data relevant for given adverse effect. An AOP mechanistically links the molecular initiating event (MIE) of a chemical with its biological target, a series of key events (KEs) at multiple biological levels of organization and the adverse outcome (AO, adverse effect) by the causal relationships and weight of evidence (Ankley et al., 2010). An AOP of EcR agonism leading to incomplete ecdysis associated mortality has recently been developed for arthropods (Song et al., 2017). In this AOP, it is suggested that hyperactivation the EcR may inhibit the ecdysis (molting) behavior, thus causing failure in the separation of old and new cuticle and leading to mortality. Based on this mechanistic model, one may hypothesize that EcR activation can also lead to reduced release of chitobiase to the external environment as a result of failed molting. Although the chemically-mediated EcR agonism, internal and external chitobiase activity, and molting disruption have been individually studied, the causal linkages between these events are not clear. Effort to develop and evaluate multiple bioassay and provide causal relationships that support populating the AOP with relevant data is thus currently a key challenge to the development of the AOP itself and provision of tools that may assist Integrated Approaches to Testing and Assessment (IATA) for the hazard assessment of EDCs (Tollefsen et al., 2014).

The aim of the present study was therefore to characterize the quantitative relationships between the release of chitobiase, molting frequency and EcR activation in the Cladoceran 
waterflea Daphnia magna after short-term (96h) exposure to the EcR agonist 20E. This freshwater crustacean has been used as a standard OECD (http://www.oecd.org/) toxicity testing species and demonstrated to be a good model for studying the effects of environmental endocrine disrupting chemicals (EDCs) on molting (Duchet et al., 2011; Sumiya et al., 2014; Song et al., 2016; Sumiya et al., 2016). Exposure to EcR agonists and chitin synthesis inhibitors have both been shown to inhibit molting in D. magna (Sumiya et al., 2014). Reduced release of chitobiase was also reported in D. magna exposed to the chitin synthesis inhibitor diflubenzuron (Duchet et al., 2011). Based on the knowledge, this study also aims to assess the sensitivity and suitability of ambient chitobiase activity as an indicator of molting disruption in D. magna.

\section{Materials and Methods}

\section{Culture}

Daphnia magna (DHI strain) were originally obtained from DHI Water Environment Health (Hørsholm, Denmark) and have been cultured in the laboratory of the Norwegian Institute for Water Research (NIVA) for more than 10 years. Briefly, 20 female D. magna were cultured in $1000 \mathrm{~mL}$ M7 medium (OECD Test Guideline 211) in each glass beaker (animal load: $50 \mathrm{~mL}$ medium/daphnid) under conditions of $\mathrm{pH} 7.8 \pm 0.2$, temperature $20 \pm 1{ }^{\circ} \mathrm{C}$ and photoperiod $16 \mathrm{~h}: 8 \mathrm{~h}$. The culture medium was renewed twice a week. Daphnids were daily fed with concentrated unicellular green algae Pseudokirchneriella subcapitata, corresponding to approximately 0.1 mg carbon daphnid ${ }^{-1}$ day $^{-1}$. 


\section{Exposure}

20-Hydroxyecdysone (CAS 5289-74-7, purity $>98 \%$ ) was purchased from Abcam (Cambridge, UK) and dissolved in dimethyl sulfide (DMSO, Sigma-Aldrich, St. Louis, USA). Exposure was conducted based on the basic principles of the Daphnia sp. Acute Immobilisation Test (OECD Test Guideline 202). Briefly, neonatal ( $<24 \mathrm{~h}$ old) D. magna were exposed to seven nominal concentrations $(15.6,31.2,62.5,125,250,500$ and $1000 \mathrm{nM})$ of $20 \mathrm{E}$ and the solvent (DMSO) control for $96 \mathrm{~h}$. The solvent concentration was $0.01 \%$ in all groups. The test was run in quadruplicate (each containing 10 daphnids in $50 \mathrm{~mL}$ exposure medium in a $100 \mathrm{~mL}$ glass beaker). The exposure medium was renewed once after $48 \mathrm{~h}$. The test animals were fed daily with $P$. subcapitata to avoid potential stress from starvation. Survival, molting frequency (number of molts) and lethal incomplete ecdysis (old cuticle still attached to the new cuticle at death) were recorded daily. The exuvia and dead daphnids were removed after observation to avoid potential aggregation of microorganisms in the exposure medium. The exposure medium $(1.5 \mathrm{~mL})$ was sampled every $24 \mathrm{~h}$ by filtering through a sterile $0.2 \mu \mathrm{m}$ polycarbonate filter (Millipore Corp., Molsheim, France) using a sterile syringe to remove bacteria and algae, and stored at $4^{\circ} \mathrm{C}$ prior to chitobiase analysis.

\section{Chitobiase analysis}

The chitobiase activity in the exposure medium was determined according to the methods described previously (Sastri and Roff, 2000b; Hanson and Lagadic, 2005). Briefly, 4methylumbelliferyl $N$-acetyl- $\beta$-D-glucosaminide (MUF-NAG, Sigma-Aldrich) was used as a 
substrate. This substrate can be cleaved into NAG and fluorescent 4-methylumbelliferone (MUF) by chitobiase and the fluorescent intensity of MUF is thus proportional to the activity of chitobiase. The stock solution of MUF-NAG ( $5 \mathrm{mM})$ was made by dissolving the chemical in methylcellosolve (Sigma-Aldrich). The medium samples $(150 \mu \mathrm{L}, \mathrm{N}=4)$ were incubated with $0.31 \mathrm{mM}$ MUF-NAG (diluted in $50 \mu \mathrm{L} 0.15 \mathrm{M}$ citrate phosphate buffer, $\mathrm{pH} 5.5$ ) for $1 \mathrm{~h}$ in Corning ${ }^{\text {TM }}$ 96-well black polystyrene microplates (Fisher Scientific, Pittsburgh, USA). The reaction was run in technical triplicates and stopped by adding $50 \mu \mathrm{L} 0.25 \mathrm{M} \mathrm{NaOH}$. The fluorescence was measured immediately using Victor 3 fluorescent plate reader (Perkin Elmer, Waltham, USA) at excitation/emission wavelength of $360 \mathrm{~nm} / 450 \mathrm{~nm}$. Five concentrations (10, $30,90,270,810 \mathrm{nM}$ ) of MUF (Sigma-Aldrich) were included in each plate for generating standard curves. The chitobiase activity was quantified based on the standard curve and expressed as nM MUF liberated per hour (nM/h). Relative chitobiase activity (\%) was calculated by comparing the exposed groups to the control and the resulting data used for statistical analysis.

\section{Two-hybrid reporter gene bioassay}

A two-hybrid in vitro screening bioassay for detection of the EcR reporter gene activation in $D$. magna was performed according to Kato et al. (2007) with some modifications (Song et al., 2016). Briefly, Chinese hamster ovary (CHO-K1) cells (ECACC, Salisbury, UK) were cultured in Dulbecco's modified Eagle's medium (Gibco, Thermo Fisher Scientific, Waltham, USA) containing $10 \%$ fetal bovine serum (Sigma-Aldrich) at $37^{\circ} \mathrm{C}$ and $5 \% \mathrm{CO}_{2}$. The cells were transferred to a 24-well plate (10000 cells/well) and transfected for 4h using FuGENE6 (Roche 
Diagnostics, Basel, Switzerland) following the original protocol from the manufacturer. In the transfection mixture, $30 \mathrm{ng}$ pBIND-dapEcR (LBD) vector, $30 \mathrm{ng}$ pACT-dapUSP (LBD) vector, $100 \mathrm{ng}$ aACT-droTaiman (LXXLL) vector, $300 \mathrm{ng}$ pG5Luc vector and $100 \mu \mathrm{L}$ fetal bovine serum were added. The vectors were initially provided by Prof. Taisen Iguchi (National Institute for Basic Biology, NIBB, Okazaki, Japan), cloned by transforming the chemically competent Escherichia coli (Invitrogen, Carlsbad, California, USA), and purified using GenElute ${ }^{\mathrm{TM}}$ HP Plasmid Midiprep Kit (Sigma-Aldrich), according to the manufacturer's instructions. The ecdysone steroid hormone ponasterone A (Wako Pure Chemical Industries Ltd., Osaka, Japan) was used as the positive control at $1000 \mathrm{nM}$ and DMSO used as the negative control. The concentration of 20E ranged from $0.1-1000 \mathrm{nM}$. The transfected cells were exposed to the hormone analogs for $40 \mathrm{~h}$ at $37^{\circ} \mathrm{C}$ and the procedure was run in technical triplicates. The activity of the reporter gene was determined using Dual-Luciferase ${ }^{\circledR}$ Reporter Assay System Kit (Promega, Madison, USA). The luminescence intensity was measured using Victor 3 Multilabel Plate Reader (Perkin Elmer). The assay was repeated independently for three times. The median expression of the technical replicates in each independent study was calculated and used as biological replicates $(\mathrm{N}=3)$. Relative expression was scaled between 0 (negative control) and $100 \%$ (maximal induction, $1000 \mathrm{nM}$ ponasterone A) prior to statistical analysis.

\section{Statistical analysis}

Data were checked for normality and equal variance prior to statistical analysis of differences between control and exposed animals using one-way analysis of variance (one-way ANOVA) followed by Tukey post-hoc test, or Kruskal-Wallis non-parametric test (no normality or equal 
variance) followed by Dunn's posthoc test. The statistical analyses were performed in Graphpad Prism v6.01 (Graphpad Software Inc., La Jolla, USA) and a probability ( $p$ ) value of 0.05 was applied to all analyses. The median lethal (LC50) and effect (EC50) concentrations were estimated based on a sigmoidal concentration-response curve (variable slope) calculated using Graphpad.

\section{Results}

The EcR reporter gene assay showed that exposure to as low as $100 \mathrm{nM} 20 \mathrm{E}$ may marginally induce the reporter gene expression, whereas exposure to $1000 \mathrm{nM} 20 \mathrm{E}$ caused approximately $25 \%$ activation of EcR in vitro compared to the positive control (Figure 1).

Results from the chitobiase analysis showed that the ambient chitobiase activity in the control media increased with the test duration, and coincided with the molting events that occurred in D. magna (Figure 2A). Exposure to $20 \mathrm{E}$ reduced the ambient chitobiase activity in a concentration-dependent manner, with exposure to $1000 \mathrm{nM} 20 \mathrm{E}$ significantly reducing chitobiase activity after $24 \mathrm{~h}(p=0.0008), 125 \mathrm{nM}$ and higher concentrations of $20 \mathrm{E}$ significantly decreasing the chitobiase activity after $72 \mathrm{~h}(p=0.0011), 250 \mathrm{nM}$ and higher concentrations of $20 \mathrm{E}$ reducing chitobiase after $96 \mathrm{~h}(p<0.0001)$. After 48h, slight but non-significant elevation of chitobiase activity was observed after exposure to 62.5 and $125 \mathrm{nM} 20 \mathrm{E}$, whereas a marginal non-significant reduction was observed at $1000 \mathrm{nM} 20 \mathrm{E}$. Based on the concentration-response curves, the estimated EC50s of 20E for chitobiase activity were 717.3 (24h), 446.7 (72h) and $397.2(96 h) \mathrm{nM}$. 
The effect of 20E on the molting frequency of D. magna was also concentration-dependent (Figure 3A), with $1000 \mathrm{nM}$ significantly reduced molting after $48 \mathrm{~h}(p=0.01), 72 \mathrm{~h}(p<0.0001)$ and 96h $(p<0.0001)$. No significant reduction in molting frequency was observed after $24 \mathrm{~h}$ exposure or at concentrations lower than $1000 \mathrm{nM}$, albeit slight, but non-significant decrease in molting frequency was apparently observed in daphnids exposed to the lowest (15.6 nM) concentration of $20 \mathrm{E}$ after 24,48 and $96 \mathrm{~h}$.

Results from the $96 \mathrm{~h}$ toxicity test showed that the lethal effect of $20 \mathrm{E}$ on the survival of $D$. magna was concentration-dependent, with exposure to $1000 \mathrm{nM} 20 \mathrm{E}$ significantly reducing the survival after $48 \mathrm{~h}(p<0.0001), 72 \mathrm{~h}(p<0.0001)$ and $96 \mathrm{~h}(p<0.0001)$. After $24 \mathrm{~h}$, the highest concentration of $20 \mathrm{E}$ slightly decreased the survival of D. magna, however, this reduction was not significant. Marginal and non-significant lethal effects were also observed in D. magna after 48, 72 and $96 \mathrm{~h}$ exposure to $500 \mathrm{nM} 20 \mathrm{E}$. Exposure to lower concentrations of 20E did not lead to any lethality. Based on the concentration-response curves (Figure 3B), the estimated median lethal concentrations (LC50) of 20E for survival were 826.7 (72h) and 738.9 (96h) nM. A summary of estimated effect concentrations for different endpoints is shown in Table 1. In addition, lethal incomplete ecdysis was observed in daphnids after 72 and $96 \mathrm{~h}$ exposure to 1000 nM 20E (Figure 3C).

\section{Discussion}

The present study mainly focused on using the release of chitobiase as an indicator of molting disruption in D. magna after short-term exposure to the endogenous EcR agonist 20E. The 
chitobiase activities detected in the exposure medium were in the same order of magnitude as that reported for D. magna previously (Duchet et al., 2011; Qi et al., 2013), confirming the validity of the bioassay for determining exogenous enrichment of this molting indicator. Moreover, temporal changes of chitobiase activity in the controls (unexposed) were similar to that observed by Duchet et al. (2011) and Qi et al. (2013), who also used $<24 \mathrm{~h}$ old neonatal $D$. magna at the start of the exposure and found increased chitobiase release from 24 to $96 \mathrm{~h}$. In the present studies, a temporal increase in the release of chitobiase to the ambient medium was observed in unexposed daphnids, whereas a concentration-dependent decrease in the release of chitobiase was observed after exposure to 20E. Based on the concentration-response curves, as low as $250 \mathrm{nM} 20 \mathrm{E}$ was found to significantly reduce the chitobiase release in D. magna after $72 \mathrm{~h}$, thus suggesting $72 \mathrm{~h}$ to be optimal for studying the effect of $20 \mathrm{E}$ on chitobiase release. Although not tested specifically herein, chitobiase may be caused by other sources than the molting in D. magna such as microorganisms (Conley et al., 2009) and may potentially contribute to the chitobiase activity measured during the test. Since the exposure study described herein was conducted in a highly controlled manner (i.e. all groups under the same conditions), and only relative expression of chitobiase (i.e. exposed vs control) was used, it is considered that the influence from the microorganisms was minimized.

In comparison with molting, the temporal release of chitobiase was generally in accordance with the molting frequency in unexposed (control) animals. The activity of chitobiase in unexposed daphnids generally increased during the test period, possibly indicating that the growth (size) of the animal may also influence the ambient activity of chitobiase, as previously demonstrated (Conley et al., 2009; Duchet et al., 2011). 
Results from the EcR reporter assay in the present study suggested that exposure to $1000 \mathrm{nM}$ 20E in vitro significantly induce the EcR expression. This effect concentration was similar to that reported by Kato et al. (2007). Chitobiase is considered under direct regulation of EcR, as induction of chitobiase expression has been observed in both crustaceans and insects after exposure to EcR agonists (Reynolds and Samuels, 1996; Zou and Fingerman, 1999b; Merzendorfer and Zimoch, 2003; Zheng et al., 2008). It may be deduced from the in vitro data that the expression of chitobiase in D. magna was likely induced at similar concentrations in the present study. On the other hand, release of chitobiase to the environment may also be indicative of incomplete ecdysis as a downstream event of EcR-mediated disruption of normal molting behavior (Song et al., 2017), as the release of molting fluid is considered highly dependent on the separation of old and new cuticle at the end of a molt cycle (Reynolds and Samuels, 1996; Duchet et al., 2011). Recent effort to evaluate the causality between the hyperactivation of the EcR with triggering of key molecular and cellular events that eventually lead to molting interference and mortality, identify that processes relevant for chitobiase release are indeed key in an AOP that describe points of departure from normal endocrine function due to exposure to putative endocrine disruptors in arthropods (https://aopwiki.org/aops/4).

When comparing the chitobiase activity with the molting frequency in animals exposed to $20 \mathrm{E}$, concordance was only found at $1000 \mathrm{nM} 20 \mathrm{E}$, where significant reduction in ambient chitobiase and molting were observed after 72 and $96 \mathrm{~h}$ exposure. However, at this exposure concentration, severe lethal effects were also observed after 72 and 96h, thus making it difficult to judge whether these ED effects were consequences of lethality, or vice versa. It was likely that the lethal effect of $20 \mathrm{E}$ was attributed by impaired molting, as incomplete ecdysis was 
observed in animals at death in the present study. Unsuccessful exuviation was observed in juvenile D. magna after 2 d exposure to $1000 \mathrm{nM} 20 \mathrm{E}$ (Bodar et al., 1990). Lethal incomplete ecdysis was previously documented in juvenile D. magna after exposure to $20 \mathrm{E}(260 \mathrm{nM})$ and ponasterone A (27 nM) in a 21d chronic test (Baldwin et al., 2001). Acute (48h) exposure to $500 \mathrm{nM} 20$ E was also demonstrated to cause complete inhibition of molting in adult female $D$. magna (Sumiya et al., 2014). In addition, similar molting defects have been frequently reported in various insects after exposure to EcR agonists (Retnakaran et al., 2003; Reynolds et al., 2009; Gelbic et al., 2011). These evidences taken together suggest that the ED effects of 20E on molting were likely the causes of lethality.

This hypothesis was further supported by significant reduction in chitobiase release observed at sublethal concentrations of $20 \mathrm{E}$ (e.g. 250 and $500 \mathrm{nM}$ after $72 \mathrm{~h}, 500 \mathrm{nM}$ after $96 \mathrm{~h}$ ). Interestingly, at these concentrations, no significant decrease in molting frequency was observed, indicating that physiological changes such as activation of EcR and chitobiase occurred at lower concentrations than those causing adverse effects on molting. The present study suggested that changes in chitobiase activity was likely not the only physiological change required to cause molting disturbance. This agree well with suggestions that activation of the EcR, inhibition of neuropeptide release, reduced neurotransmission and ecdysis motor program, and incomplete separation of old and new cuticles ultimately lead to molting disruption (Song et al., 2017). Although causal relationships between EcR activation, reduction in chitobiase and perturbations of normal molting is proposed herein, better synchronization of the test animals at the onset of studies may reduce variability and increase the sensitivity of the assays used. The duration of the molt cycle may serve as a sensitive endpoint for disturbance of normal 
molting and thus be used to assess causality between molting quality and release of chitobiase, as in many cases, delayed molting may occur rather than complete inhibition of molting. Slight delay in molting may not influence the overall molting frequency when observations are made at time intervals longer than the molt cycle itself. This in particular applies to test with neonatal D. magna which normally perform the first and second juvenile molting at approximately 27.5 and 52h after birth (Mu and LeBlanc, 2004). It was clearly shown that the internal (tissue) activity of chitobiase negatively correlated with the duration of molt cycle in $D$. magna under food or temperature stress (Espie and Roff, 1995). The linkage between the release of chitobiase to the external environment and duration of molt cycles, however, has not been studied in detail yet.

Furthermore, when comparing the effect concentrations of $20 \mathrm{E}$ between different endpoints (Table 1), the release of chitobiase was identified to be the most sensitive endpoint in response to $20 \mathrm{E}$, especially after 72 and $96 \mathrm{~h}$. The LOEC obtained from the current study suggested that chitobiase was the most sensitive indicator of changes to ecdysis. Induction of the EcR may likely occur at similar concentrations, although the experimental design of the in vitro EcR activation study did not allow sufficient discriminating power to assess this in detail. It is still not clear how reduction in chitobiase release correlates with the activation of the EcR in vivo, as both molting quality and other factors such as size of the animal may potentially influence the ambient chitobiase activity. Future studies should aim at determining the endogenous chitobiase mRNA and enzyme expression after exposure to EcR agonists, and causally as well as quantitatively link the responses to the duration of molt cycles and ambient chitobiase activities. In addition, the approaches described herein need to be further verified using other 
environmental EcR agonists (e.g. pesticides) to evaluate the assays suitability for screen environmentally relevant EDCs. Knowledge generated from such studies may be used to develop the chitobiase assay as a screening tool for chemicals with EcR agonistic and molting disrupting activities, and by doing so assist screening and prioritization of chemicals, limiting experimental animal testing as part of IATA approaches in assessment of EDCs (Tollefsen et al., 2014).

\section{Conclusions}

The present study evaluated the applicability of using chitobiase release as an indicator of potential endocrine disruption of molting in juvenile D. magna after short-term exposure to the model EcR agonist 20E. The results clearly showed that the release of chitobiase was more sensitive than other relevant endpoints such as molting frequency and survival, and may together with activation of the EcR be used as a biomarker for endocrine disrupting chemicals that interfere with EcR-mediated toxicity pathways leading to molting disturbance. As a first attempt to identify linkages between EcR agonism, molting and chitobiase release, the present study provides an initial assessment of the suitability of these endpoints in ED studies focusing on molting and development in arthropods.

\section{Acknowledgements}

The present project was funded by Research Council of Norway, project NFR-221455 "Adverse 
Outcome Pathways for Endocrine Disruption in Daphnia magna, a Conceptual Approach for Mechanistically-based Risk Assessment (EDRISK)". The authors would like to acknowledge Hitoshi Miyakawa (NIBB), Li Xie, Tânia Gomes, David Eidsvoll and Maria T. Hultman (NIVA) for their contribution to the experimental work, and appreciate the advices from Adam Lillicrap and Ailbhe Macken (NIVA).

\section{References}

Ankley, G.T., Bennett, R.S., Erickson, R.J., Hoff, D.J., Hornung, M.W., Johnson, R.D., Mount, D.R., Nichols, J.W., Russom, C.L., Schmieder, P.K., Serrrano, J.A., Tietge, J.E., Villeneuve, D.L., 2010. Adverse outcome pathways: a conceptual framework to support ecotoxicology research and risk assessment. Environ. Toxicol. Chem. 29, 730-741.

Baldwin, W.S., Bailey, R., Long, K.E., Klaine, S., 2001. Incomplete ecdysis is an indicator of ecdysteroid exposure in Daphnia magna. Environ. Toxicol. Chem. 20, 1564-1569.

Bodar, C.W.M., Voogt, P.A., Zandee, D.I., 1990. Ecdysteroids in Daphnia magna: Their role in molting and reproduction and their levels upon exposure to cadmium. Aquat. Toxicol. 17, 339-350.

Conley, J.M., Schorr, M.S., Hanson, M.L., Symes, S.J., Richards, S.M., 2009. Is ambient chitobiase activity a monitoring tool for impacts on secondary production in lotic systems? Can. J. Fish. Aquat. Sci. 66, 1274-1281.

de Souza Machado, A.A., Zarfl, C., Rehse, S., Kloas, W., 2017. Low-dose effects: nonmonotonic responses for the toxicity of a Bacillus thuringiensis biocide to Daphnia magna. Environ. Sci. Technol. In press.

De Wilde, R., Swevers, L., Soin, T., Christiaens, O., Rouge, P., Cooreman, K., Janssen, C.R., Smagghe, G., 2013. Cloning and functional analysis of the ecdysteroid receptor complex in the opossum shrimp Neomysis integer (Leach, 1814). Aquat. Toxicol. $130-131,31-40$.

Duchet, C., Mitie Inafuku, M., Caquet, T., Larroque, M., Franquet, E., Lagneau, C., Lagadic, L., 2011. Chitobiase activity as an indicator of altered survival, growth and reproduction in Daphnia pulex and Daphnia magna (Crustacea: Cladocera) exposed to spinosad and diflubenzuron. Ecotoxicol. Environ. Saf. 74, 800-810.

Espie, P.J., Roff, J.C., 1995. A biochemical index of duration of the molt cycle for planktonic crustacea based on the chitindegrading enzyme, chitobiase. Limnol. Oceanogr. 40, 1028-1034.

Gelbic, I., Adel, M.M., Hussein, H.M., 2011. Effects of nonsteroidal ecdysone agonist RH-5992 and chitin biosynthesis inhibitor lufenuron on Spodoptera littoralis (Boisduval, 1833). Cent. Eur. J. Biol. 6, 861-869.

Hanson, M., Lagadic, L., 2005. Chitobiase activity as an indicator of aquatic ecosystem health. Aquat. Ecosyst. Health Manage. 8, $441-450$ 
Kato, Y., Kobayashi, K., Oda, S., Tatarazako, N., Watanabe, H., Iguchi, T., 2007. Cloning and characterization of the ecdysone receptor and ultraspiracle protein from the water flea Daphnia magna. J. Endocrinol. 193, 183-194.

Meng, Y.L., Zou, E.M., 2009. A molecular biomarker for disruption of crustacean molting: the $N$-acetyl-beta-glucosaminidase mRNA in the epidermis of the fiddler crab. Bull. Environ. Contam. Toxicol. 82, 554-558.

Merzendorfer, H., Zimoch, L., 2003. Chitin metabolism in insects: structure, function and regulation of chitin synthases and chitinases. J. Exp. Biol. 206, 4393-4412.

$\mathrm{Mu}, \mathrm{X}$., LeBlanc, G.A., 2004. Synergistic interaction of endocrine-disrupting chemicals: model development using an ecdysone receptor antagonist and a hormone synthesis inhibitor. Environ. Toxicol. Chem. 23, 1085-1091.

Nakagawa, Y., 2005. Nonsteroidal ecdysone agonists. Vitam. Horm. 73, 131-173.

NRC, 2007. Toxicity Testing in the 21st Century: A Vision and a Strategy. National Research Council.

Qi, S., Wang, C., Chen, X., Qin, Z., Li, X., Wang, C., 2013. Toxicity assessments with Daphnia magna of Guadipyr, a new neonicotinoid insecticide and studies of its effect on acetylcholinesterase (AChE), glutathione S-transferase (GST), catalase (CAT) and chitobiase activities. Ecotoxicol. Environ. Saf. 98, 339-344.

Retnakaran, A., Krell, P., Feng, Q.L., Arif, B., 2003. Ecdysone agonists: mechanism and importance in controlling insect pests of agriculture and forestry. Arch. Insect Biochem. 54, 187-199.

Reynolds, S.E., 1987. The cuticle, growth and molting in insects: the essential background to the action of acylurea insecticides. Pestic. Sci. 20, 131-146.

Reynolds, S.E., Brown, A.M., Seth, R.K., Riddiford, L.M., Hiruma, K., 2009. Induction of supernumerary larval moulting in the tobacco hornworm Manduca sexta: interaction of bisacylhydrazine ecdysteroid agonists with endogenous juvenile hormone. Physiol. Entomol. 34, 30-38.

Reynolds, S.E., Samuels, R.I., 1996. Physiology and biochemistry of insect moulting fluid. Adv. Insect Physiol. 26, 157-232.

Richards, S.M., Kelly, S.E., Hanson, M.L., 2008. Zooplankton chitobiase activity as an endpoint of pharmaceutical effect. Arch. Environ. Contam. Toxicol. 54, 637-644.

Sastri, A.R., Roff, J.C., 2000a. Rate of chitobiase degradation as a measure of development rate in planktonic Crustacea. Can J Fish Aquat Sci 57, 1965-1968.

Sastri, A.R., Roff, J.C., 2000b. Rate of chitobiase degradation as a measure of development rate in planktonic Crustacea. Can J Fish Aquat Sci 57, 1965-1968.

Song, Y., Rundberget, J.T., Evenseth, L.M., Xie, L., Gomes, T., Hogasen, T., Iguchi, T., Tollefsen, K.E., 2016. Whole-organism transcriptomic analysis provides mechanistic insight into the acute toxicity of emamectin benzoate in Daphnia magna. Environ. Sci. Technol. 50, 11994-12003.

Song, Y., Villeneuve, D.L., Toyota, K., Iguchi, T., Tollefsen, K.E., 2017. Ecdysone receptor agonism leading to lethal molting disruption in arthropods: Review and adverse outcome pathway development. Environ Sci Technol 51, 4142-4157. 
Spindler-Barth, M., Van Wormhoudt, A., Spindler, K.-D., 1990. Chitinolytic enzymes in the integument and midgut-gland of the shrimp Palaemon serratus during the moulting cycle. Mar. Biol. 106, 49-52.

Sumiya, E., Ogino, Y., Miyakawa, H., Hiruta, C., Toyota, K., Miyagawa, S., Iguchi, T., 2014. Roles of ecdysteroids for progression of reproductive cycle in the fresh water crustacean Daphnia magna. Front. Zool. 11, 60.

Sumiya, E., Ogino, Y., Toyota, K., Miyakawa, H., Miyagawa, S., Iguchi, T., 2016. Neverland regulates embryonic moltings through the regulation of ecdysteroid synthesis in the water flea Daphnia magna, and may thus act as a target for chemical disruption of molting. J. Appl. Toxicol. 36, 1476-1485.

Tollefsen, K.E., Scholz, S., Cronin, M.T., Edwards, S.W., de Knecht, J., Crofton, K., Garcia-Reyero, N., Hartung, T., Worth, A., Patlewicz, G., 2014. Applying Adverse Outcome Pathways (AOPs) to support Integrated Approaches to Testing and Assessment (IATA). Regul. Toxicol. Pharmacol. 70, 629-640.

Zheng, Y.P., Krell, P.J., Doucet, D., Arif, B.M., Feng, Q.L., 2008. Cloning, expression, and localization of a molt-related beta- $N$ acetylglucosaminidase in the spruce budworm, Choristoneura fumiferana. Arch. Insect Biochem. Physiol. 68, 49-59.

Zitnan, D., Kim, Y.J., Zitnanova, I., Roller, L., Adams, M.E., 2007. Complex steroid-peptide-receptor cascade controls insect ecdysis. Gen. Comp. Endocrinol. 153, 88-96.

Zou, E., Fingerman, M., 1999a. Chitobiase activity in the epidermis and hepatopancreas of the fiddler crab Uca pugilator during the molting cycle. Mar. Biol. 133, 97-101.

Zou, E., Fingerman, M., 1999b. Patterns of N-acetyl-beta-glucosaminidase isoenzymes in the epidermis and hepatopancreas and induction of $N$-acetyl-beta-glucosaminidase activity by 20-hydroxyecdysone in the fiddler crab, Uca pugilator. Comp. Biochem. Physiol. C Pharmacol. Toxicol. Endocrinol. 124, 345-349.

Zou, E.M., 2005. Impacts of xenobiotics on crustacean molting: the invisible endocrine disruption. Integrat. Comp. Biol. 45, 3338 
Table 1. A summary of effect concentrations (nmol/L) of 20-hydroxyecdysone for different endpoints in juvenile Daphnia magna.

\begin{tabular}{|c|c|c|c|c|c|c|c|c|c|c|c|c|c|c|c|}
\hline Endpoint & & 24h & & & $48 \mathrm{~h}$ & & & $72 \mathrm{~h}$ & & & $96 \mathrm{~h}$ & & & in vitro & \\
\hline & NOEC & LOEC $^{\mathrm{b}}$ & EC50 & NOEC & LOEC & EC50 & NOEC & LOEC & EC50 & NOEC & LOEC & EC50 & NOEC & LOEC & EC50 \\
\hline Survival & 1000 & $>1000$ & $1348^{\circ}$ & 500 & 1000 & $1520^{\circ}$ & 500 & 1000 & 826.7 & 500 & 1000 & 738.9 & I & I & I \\
\hline Molting & 1000 & $>1000$ & $2801^{\circ}$ & 500 & 1000 & $1489^{\circ}$ & 500 & 1000 & $1776^{\circ}$ & 500 & 1000 & $1478^{\circ}$ & I & I & ' \\
\hline Chitobiase & 500 & 1000 & 717.3 & 1000 & $>1000$ & $3730^{\circ}$ & 125 & 250 & 446.7 & 250 & 500 & 397.2 & I & I & I \\
\hline $\mathbf{E c R}^{\mathrm{d}}$ & 1 & 1 & I & 1 & 1 & 1 & I & 1 & 1 & 1 & 1 & 1 & 100 & 1000 & $2153^{\mathrm{e}}$ \\
\hline
\end{tabular}

a. NOEC: No Observed Effect Concentration

b. LOEC: Lowest Observed Effect Concentration

c. EC50: Median Effect Concentration

d. EcR: Ecdysone receptor activity

e. Predicted values, observed effect less than $50 \%$ 
Figures:

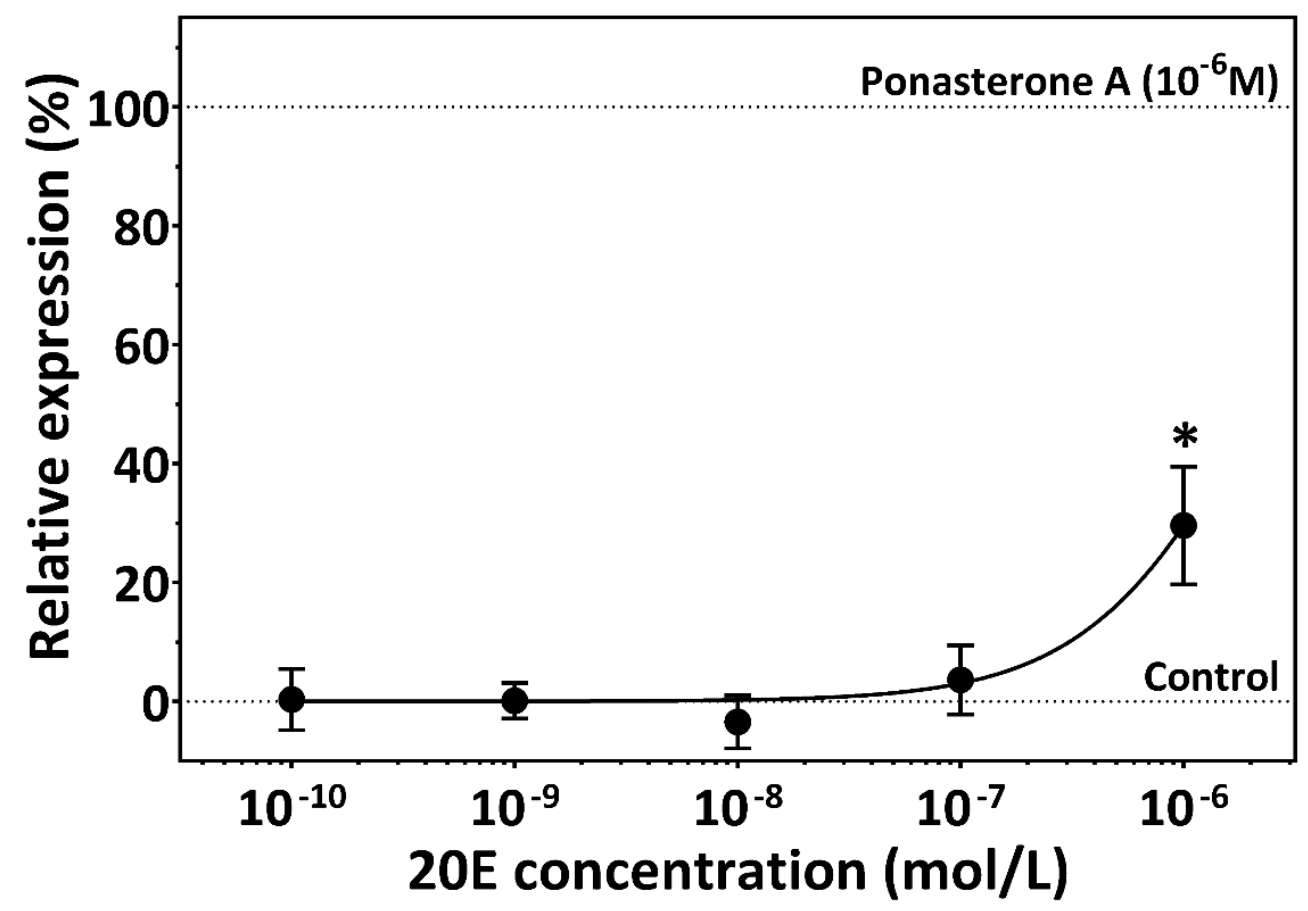

Figure 1. Relative expression of Daphnia magna ecdysone receptor (EcR) in transfected Chinese hamster ovary (CHO-K1) cells after 40h exposure to 0.1-1000 nM 20-hydroxyecdysone (20E). The relative expression was scaled between the expression of EcR in the control (DMSO, $0 \%$ induction) and positive control (1000 nM ponasterone A, $100 \%$ induction). Results are presented as mean $\pm \mathrm{SEM}, \mathrm{N}=3 . *$ denotes significant different from the control $(p=0.0224)$.
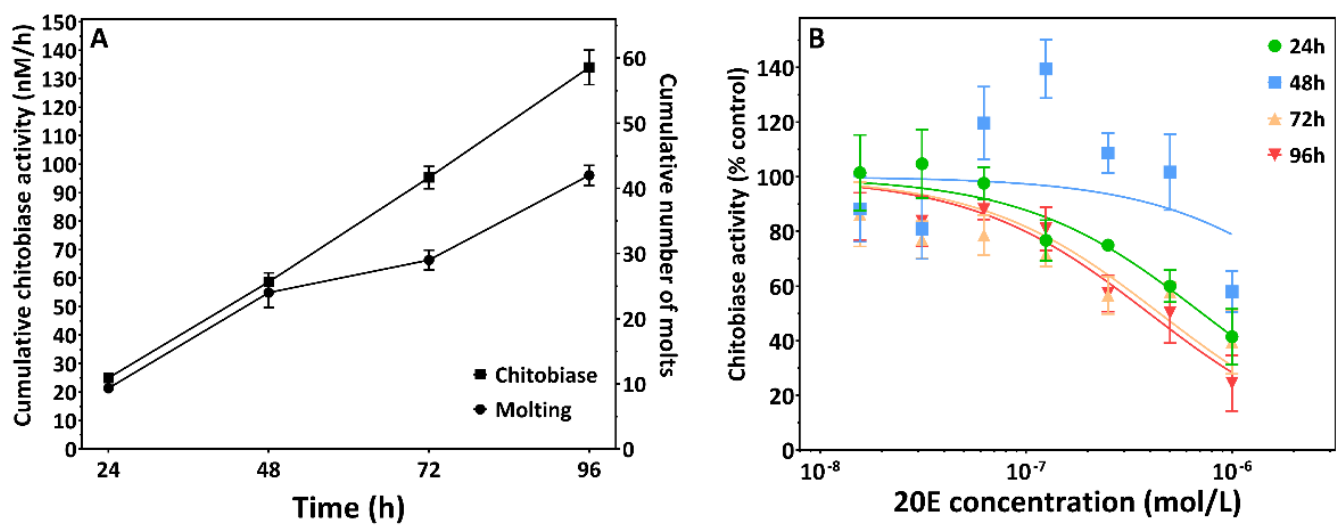
Figure 2. Temporal (24-96h) change of cumulative chitobiase activity and cumulative molting frequency in unexposed (control) juvenile Daphnia magna (A); Effects of 20-hydroxyecdysone on chitobiase release in D. magna after 24-96h exposure (B). Results are presented as mean $\pm \mathrm{SEM}, \mathrm{N}=4$ (10 individuals in each replicate).

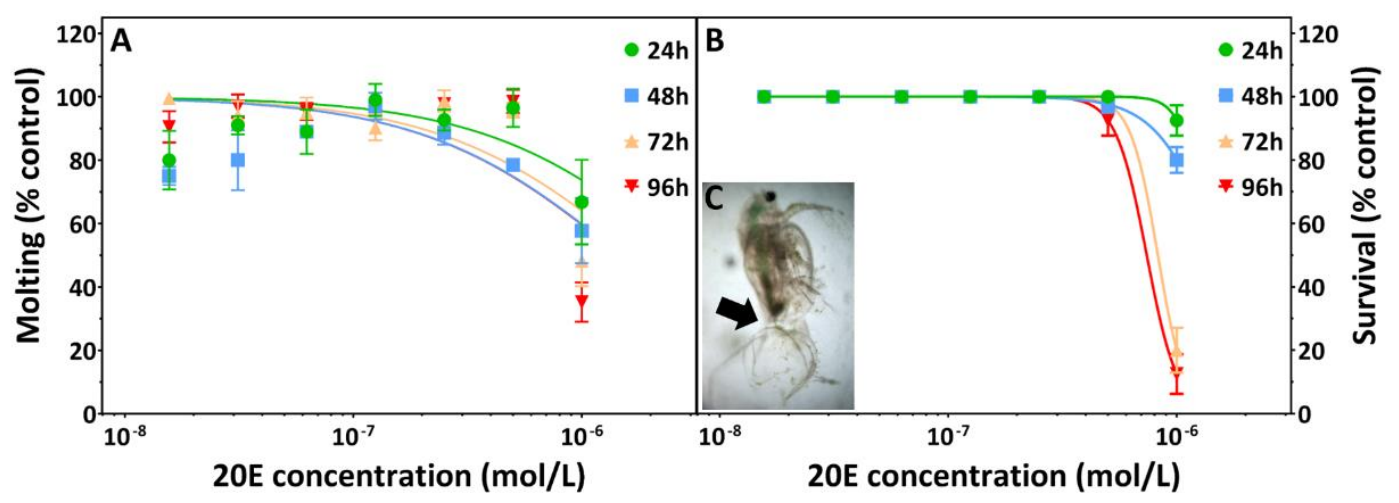

Figure 3. Molting frequency (A) and survival (B) in juvenile Daphnia magna after 24-96h exposure to 20-hydroxyecdysone (20E), results are shown in \% compared to the control and presented as mean $\pm \mathrm{SEM}$, $\mathrm{N}=4$; Lethal incomplete ecdysis in D. magna after $72 \mathrm{~h}$ exposure to $1000 \mathrm{nM} 20 \mathrm{E}$ (C), arrow indicates incomplete shedding of old cuticle in D. magna at death. 